\title{
Salivary molecules of bone remodeling and tissue repair after head and neck radiotherapy
}

\section{Eduardo Morato de OLIVEIRA ${ }^{(a)}$ \\ Rafael Soares GUIEIRO(a) \\ Bárbara Lima CRUZ(a) \\ Maria Cássia Ferreira de AGUIAR(a) \\ Janine Mayra da SILVA(a) \\ Tarcília Aparecida SILVA(a) \\ Patrícia Carlos CALDEIRA(a) (D)}

(a) Universidade Federal de Minas Gerais UFMG, School of Dentistry, Department of Oral Surgery and Pathology, Belo Horizonte, MG, Brazil.

Declaration of Interests: The authors certify that they have no commercial or associative interest that represents a conflict of interest in connection with the manuscript.

\section{Corresponding Author:}

Patrícia Carlos Caldeira

E-mail: pccaldeira@ufmg.br

hitps://doi.org/10.1590/1807-3107bor-2021.vol35.0079

Submitted: October 12, 2020

Accepted for publication: February 3, 2021

Last revision: March 2, 2021
Abstract: Head and neck radiotherapy causes quantitative and qualitative changes in saliva. The objective of this case-control study was to evaluate the salivary biomarkers associated with bone remodeling and tissue repair in patients submitted to radiotherapy for head and neck cancer treatment, compared with non-irradiated individuals. Total unstimulated saliva was collected for ELISA assay analysis of receptor activator for nuclear factor $\kappa$ B (RANK) and its ligand (RANK-L), osteoprotegerin, matrix metalloproteinase-9/ tissue inhibitor of metalloproteinase-2, vascular endothelial growth factor, and epidermal growth factor. Statistics were performed, and revealed that salivary RANK $(p=0.0304)$, RANK-L $(p=0.0005)$, matrix metalloproteinase- 9 / tissue inhibitor of metalloproteinase-2 $(p=0.0067)$, vascular endothelial growth factor $(p=0.0060)$, and epidermal growth factor $(p<0.0001)$ were reduced in patients, compared with the control group. Osteoprotegerin did not differ between the groups $(p=0.3765)$. Salivary biomarkers did not differ according to radiotherapy completion time $(p>0.05)$. In conclusion, the lower output of the salivary molecules - essential for bone remodeling and tissue repair - may disrupt tissue homeostasis and play a role in the pathogenesis of the radiotherapy-induced deleterious effects in the oral cavity.

Keywords: Saliva. Radiotherapy. Bone remodeling. Mouth neoplasms. Head and neck neoplasms.

\section{Introduction}

Radiotherapy (RT) is frequently applied to treat head and neck cancer patients, in association with either surgery or chemotherapy. ${ }^{1}$ Head and neck RT is associated with early and late deleterious effects on oral health, such as hyposalivation, mucositis, tissue atrophy, and osteoradionecrosis of the jaws. $2,3,4,5,6$

The transmembrane receptor activator for nuclear factor $\kappa \mathrm{B}$ (RANK) is expressed on osteoclast cells, and induces activation and differentiation of these cells when it binds with its ligand (RANK-L) ${ }^{7,8}$ Osteoprotegerin (OPG) is a competing ligand to RANK, and acts by inhibiting osteoclast activation. ${ }^{8}$ The RANK/ RANK-L/ OPG balance is pivotal to secure proper bone remodeling. ${ }^{7}$ Matrix metalloproteinases 
are endopeptidases responsible for extracellular matrix degradation. ${ }^{9}$ MMP-9 plays an important role in bone maintenance and remodeling, and is required for the collagen-driven migration of osteoclasts and pre-osteoclasts in the long bones. ${ }^{10}$ Tissue inhibitor of metalloproteinases 2 (TIMP-2) is the natural inhibitor of matrix metalloproteinases, including MMP-9.

The vascular endothelial growth factor (VEGF) plays a major role in the proliferation, migration and activation of endothelial cells, and in promoting blood vessel permeability. ${ }^{11,12}$ Epidermal growth factor (EGF) is a single chain polypeptide ${ }^{13}$ that binds to its membrane receptor (EGFR), and has mitogenic effects on most epithelial cells, fibroblasts, and endothelial cells. ${ }^{14}$

The roles played by RANK, RANK-L, OPG, MMP-9/ TIMP-2, VEGF and EGF on hard and soft tissue homeostasis make it reasonable to hy pothesize that these biomarkers are associated with the adverse effects of head and neck RT in the oral cavity, including tissue atrophy, impaired wound healing, and osteoradionecrosis. However, most of these biomarkers have never been evaluated in the saliva of irradiated patients, and little information is available regarding their clinical application for predicting, monitoring, or prognosing oral complications of head and neck RT. ${ }^{15,16,17}$

The main goal of the current study was to assess the salivary biomarkers related to bone remodeling and tissue repair, namely RANK, RANK-L, OPG, MMP-9/ TIMP-2, VEGF, and EGF, in patients submitted to head and neck RT, compared with non-irradiated individuals. The hypothesis was that patients submitted to RT in the head and neck region would have lower output of these molecules than the controls.

\section{Methodology}

\section{Participants: patients and controls}

This is a case-control study. The study protocol was approved by the Research Ethics Committee of Universidade Federal de Minas Gerais (certificate number 85229518.9.0000.5149). All participants gave their written consent to be included in the research, conducted in accordance with the Declaration of Helsinki.

The study patients who received head and neck RT for cancer treatment were selected from the Head and Neck Cancer Patient Dental Care Clinics of the School of Dentistry, Universidade Federal de Minas Gerais, Brazil, from 2018 to 2019. The following patients were included: those who had a histologically confirmed diagnosis of malignant neoplasm of head and neck area; those who concluded cancer treatment that included RT of the head and neck region, either with or without chemotherapy; and those who had been previously submitted to an oral care protocol to control periodontal disease, caries, or foci of infection. Exclusion criteria comprised clinical signs of periodontitis (gingival bleeding or suppuration, teeth mobility, dental calculus); recurrent tumor; oro-nasal or oro-antral communication; human immunodeficiency virus or autoimmune diseases; an invasive dental procedure less than 30 days before saliva collection; use of anti-inflammatory, antibiotic, or anti-fungal drugs in the previous 30 days; previous or current use of bone-modifying agents, such as bisphosphonates; and uncontrolled systemic diseases (hypertension, diabetes mellitus). Patients whose salivary volume was insufficient for performing the proposed analyses were also excluded.

The control group was composed of healthy individuals, never diagnosed with any malignant neoplasm, or exposed to RT or chemotherapy. These individuals had been previously submitted to oral care to control periodontal disease, caries, and foci of infection. They were matched by gender and age with RT patients, and the same exclusion criteria applied to the control group. There was one control (patient) per case.

\section{Saliva collection and ELISA}

Saliva was collected from each patient at a single time point after RT. Patients were allocated into three subgroups according to their RT completion time at saliva collection: 1 to 6 months, 7 to 12 months, 13 months or more.

Whole unstimulated saliva was collected between 2 and 4 p.m. ${ }^{18}$ Prostheses were removed 
and individuals rinsed their mouth with $5 \mathrm{ml}$ of distilled water for 1 minute. The saliva produced during 5 minutes was collected in a sterile Falcon tube and placed on ice. The total volume was quantified and the saliva was centrifuged at $1500 \mathrm{rpm}$ for 10 minutes at $4^{\circ} \mathrm{C}$. Protease and phosphatase inhibitors (0.1 mmol / L of phenylmethylsulfonyl fluoride [PMSF], $0.1 \mathrm{mmol} / \mathrm{L}$ benzethonium chloride, 10 $\mathrm{mmol} / \mathrm{L}$ ethylenediaminetetraacetic acid [EDTA], $0.01 \mathrm{mg} / \mathrm{ml}$ aprotinin and $0.05 \%$ Tween 20 ) were added to the supernatant at a ratio of 1:1. The sample was stored at $-80^{\circ} \mathrm{C}$ until analysis.

RANK, OPG, MMP-9/TIMP-2, VEGF, EGF (Duo-Set ${ }^{\circledast}$, R\&D systems; Minneapolis, USA), and RANK-L (PrepoTech; Rocky Hill, NJ, SA) concentrations were assessed by enzyme-linked immunosorbent assay (ELISA), following the manufacturer's instructions. Saliva was not diluted any further. The raw concentration $(\mathrm{pg} / \mathrm{mL})$ was corrected by the salivary flow ( $\mu \mathrm{L} / \mathrm{min})$, thereby allowing the output to be expressed in $\mathrm{pg} / \mathrm{min}$.

\section{Statistical analysis}

Descriptive analysis and comparative tests were performed using SPSS ${ }^{\circledR} 19.0$ (IBM, Chicago, USA) and GraphPad Prism ${ }^{\circledast} 7.0$ (GraphPad Software, San Diego, USA) software programs. The Shapiro-Wilk test was used to evaluate the normality $(n<50)$, and the Wilcoxon test, to compare the salivary biomarker output between patients and matched controls. The Kruskal-Wallis test was used to compare the salivary biomarkers in patients according to the RT completion time at saliva collection, categorized as 1 to 6 months, 7 to 12 months, or 13 months or more. The Spearman test was used to assess the correlation between salivary biomarkers and clinical variables (sex, age, smoking, alcohol use, tumor location, T stage, N stage, TNM staging, RT dose, RT completion time, and chemotherapy) and among the salivary biomarkers themselves. The Mann-Whitney test was used to compare the salivary flow and the salivary biomarkers between patients undergoing only RT and those submitted to RT plus chemotherapy. The significance for all the tests was set at $p<0.05$.

\section{Results}

Thirty-one patients were potentially eligible; however eight were excluded due to insufficient saliva volume. Therefore, the final sample was composed of twenty-three patients, whose clinical and demographic data are shown in Table 1. Most

Table 1. Clinical and demographic characteristics of 23 patients irradiated in the head and neck region.

\begin{tabular}{|c|c|}
\hline Variable & n (\%) \\
\hline \multicolumn{2}{|l|}{ Sex } \\
\hline Male & 17 (73.9\%) \\
\hline Female & $6(26.1 \%)$ \\
\hline \multicolumn{2}{|l|}{ Smoking } \\
\hline Never & $4(17.4 \%)$ \\
\hline Yes, prior & 18 (78.3\%) \\
\hline Yes, current & $1(4.3 \%)$ \\
\hline \multicolumn{2}{|l|}{ Alcohol use } \\
\hline Never & $6(26.1 \%)$ \\
\hline Yes, prior & 12 (52.2\%) \\
\hline Yes, current & $5(21.7 \%)$ \\
\hline \multicolumn{2}{|l|}{ Tumor location } \\
\hline Mouth & 7 (30.4\%) \\
\hline Oropharynx & $8(34.8 \%)$ \\
\hline Other & $8(34.8 \%)$ \\
\hline \multicolumn{2}{|l|}{ Histological diagnosis } \\
\hline Squamous cell carcinoma & $20(87.0 \%)$ \\
\hline Lymphoma & $1(4.3 \%)$ \\
\hline Adenocarcinoma & $1(4.3 \%)$ \\
\hline Adenoid cystic carcinoma & $1(4.3 \%)$ \\
\hline \multicolumn{2}{|l|}{ T Stage } \\
\hline 1 & 2 (8.7\%) \\
\hline 2 & $5(21.7 \%)$ \\
\hline 3 & $6(26.1 \%)$ \\
\hline 4 & $8(34.8 \%)$ \\
\hline Missing & 2 (8.7\%) \\
\hline \multicolumn{2}{|l|}{ N Stage } \\
\hline 0 & 11 (47.8\%) \\
\hline 1 & $3(13.0 \%)$ \\
\hline 2 & $6(26.1 \%)$ \\
\hline 3 & $2(8.7 \%)$ \\
\hline Missing & $1(4.3 \%)$ \\
\hline
\end{tabular}


of the patients were male (73.9\%), with a mean age of 59.1 years. Lesions were mainly located in the oropharynx (30.4\%), larynx (26.1\%), and floor of the mouth $(21.7 \%)$. The mean total dose of radiation was 61.5 Gy, and the mean RT completion time was 25.8 months. Ten patients received only radiotherapy (RT-only), and thirteen received radiotherapy and chemotherapy $(\mathrm{RT}+\mathrm{CT})$. No patient showed any sign or symptoms of osteoradionecrosis when the saliva was collected.

Salivary output $(\mathrm{pg} / \mathrm{min})$ of RANK $(\mathrm{p}=0.0304)$, RANK-L ( $p=0.0005)$, MMP-9/ TIMP-2 ( $p=0.0067)$, VEGF $(p=0.0060)$, and EGF $(p<0.0001)$ was reduced in RT patients $(n=23)$, compared with the control group. OPG did not differ between the groups $(p=0.3765)$ (Figure). There was no difference in salivary flow or salivary biomarkers based on RT completion time $(p>0.05)$. The salivary flow $(\mu \mathrm{L} / \mathrm{min})$ was significantly lower in RT patients $(n=23)$ than in the controls $(\mathrm{p}=0.0009)$.

There was no strong correlation between salivary biomarkers and the clinical features analyzed (Spearman $\rho$ coefficient $<|0.7|)$. RANK-L had a strong and positive correlation with VEGF (Spearman $\rho$ coefficient $=+0.746$ ) and EGF (Spearman $\rho$ coefficient $=+0.894)$. VEGF also had a strong and positive correlation with EGF (Spearman $\rho$ coefficient $=+0.792$ ). Other associations among the salivary biomarkers showed only weak to moderate correlations (Spearman $\rho$ coefficient $<|0.7|$ ) (Table 2).

There was no difference in salivary flow between RT-only and RT+CT patients $(\mathrm{p}=0.598)$. The comparison between biomarkers in RT-only and RT $+\mathrm{CT}$ patients showed differences only in VEGF $(p=0.009)$ and MMP-9/TIMP-2 ( $p=0.032)$, with RT+CT patients showing higher values.

\section{Discussion}

The results of the current study showed a reduction in salivary flow in the irradiated patients, compared with the controls. Salivary output of RANK, RANK-L, MMP-9/ TIMP-2, VEGF, and EGF was lower in patients submitted to RT than in the controls, whereas OPG output did not differ. The lower output of these molecules may disrupt bone remodeling and tissue repair in irradiated patients, and may supposedly play a role in the pathogenesis of late deleterious effects of RT, such as osteoradionecrosis.

This is the first study to assess RANK, RANK-L, and OPG in the saliva of head and neck irradiated patients. It is known that head and neck-irradiated patients carry a higher risk of osteoradionecrosis, whose pathogenesis is related to bone hypocelularity, hypoxia and hypovascularization. ${ }^{19}$ RANK and RANK-L were reduced in the saliva of irradiated patients. This seems to indicate that the RANK/ RANK-L/ OPG axis is affected by head and neck RT, and may be an important pathway for osteoradionecrosis development. This association could not be assessed in the present study, because only two patients had a history of osteoradionecrosis. Further studies should investigate the role of RANK/ RANK-L/ OPG in osteoradionecrosis pathogenesis, and the predictive value of their salivary dosage.

Ortega et al..$^{20}$ and Paiva et al. ${ }^{10}$ reported the role of MMP-9 in the recruitment of osteoclasts and pre-osteoclasts to promote bone resorption. ${ }^{10,20}$ Nyman et al..$^{21}$ showed that the absence of MMP-9 led to an increase in trabecular density, and consequent bone fragility in the femur of mice, thus evidencing the participation of MMP-9 in maintaining the trabecular bone architecture. In addition, this enzyme participates in the wound healing process by degrading and remodeling the extracellular matrix. ${ }^{9}$ Given that MMP-9 is directly related to bone metabolism and wound healing, ${ }^{21}$ any reduced salivary output of MMP-9/ TIMP-2 in irradiated patients may also be associated with impaired bone remodeling and wound healing, thus making the alveolar jawbone and oral mucosa less responsive to injuries in irradiated patients. Indeed, the authors observed a significant reduction of this enzyme in irradiated patients, as previously reported by Oton-Leite et al. ${ }^{18}$

VEGF is mainly associated with angiogenesis and tissue repair. ${ }^{22}$ In addition, VEGF may act directly on the function of osteoblasts, by regulating their chemotaxis, proliferation, and differentiation..$^{22}$ Thus, VEGF is needed for angiogenesis and osteogenesis, and is the main molecule that correlates these two processes during bone repair. ${ }^{22,23}$ Not surprisingly, 
A

$\operatorname{RANK}(n=23)$

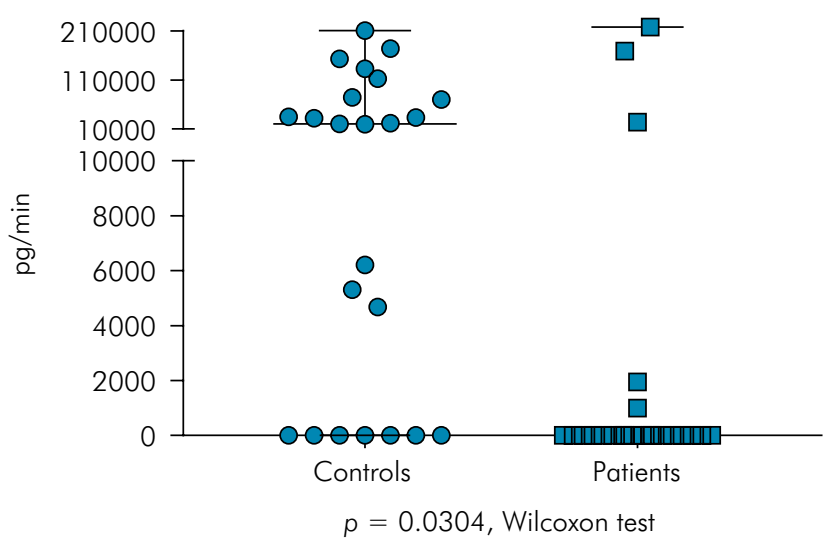

C

OPG $(n=23)$

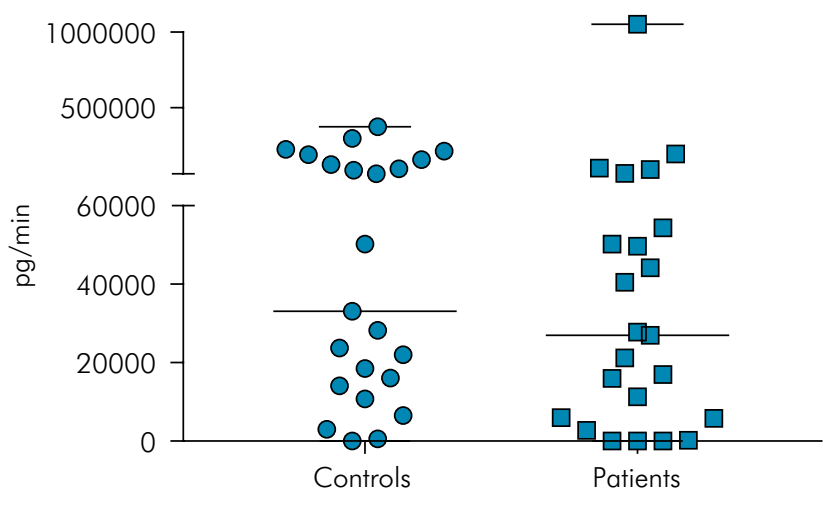

$p=0.3765$, Wilcoxon test

E

$\operatorname{VEGF}(n=23)$

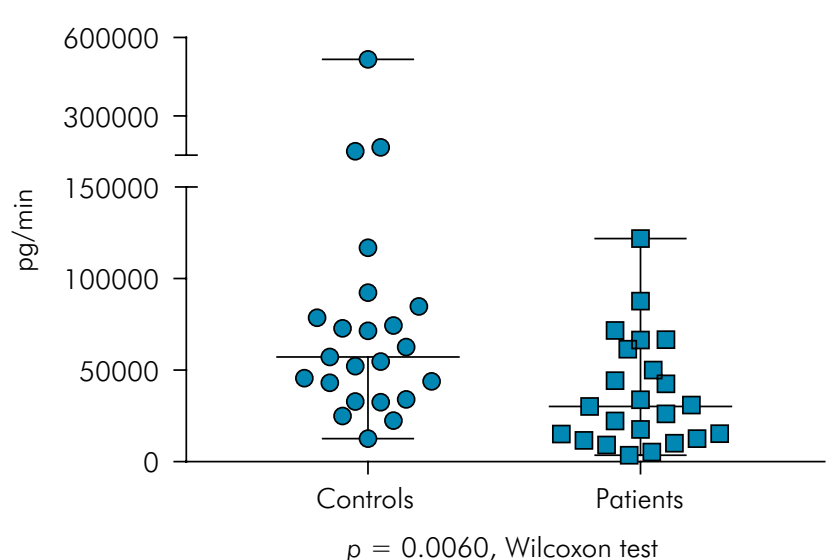

B

RANK-L $(n=23)$

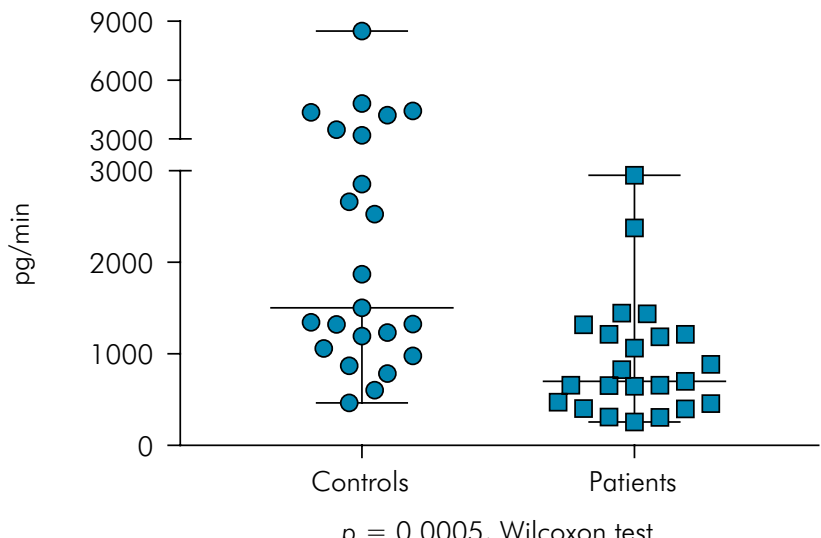

D

MMP-9/ TIMP-2 ( $\mathrm{n}=23)$

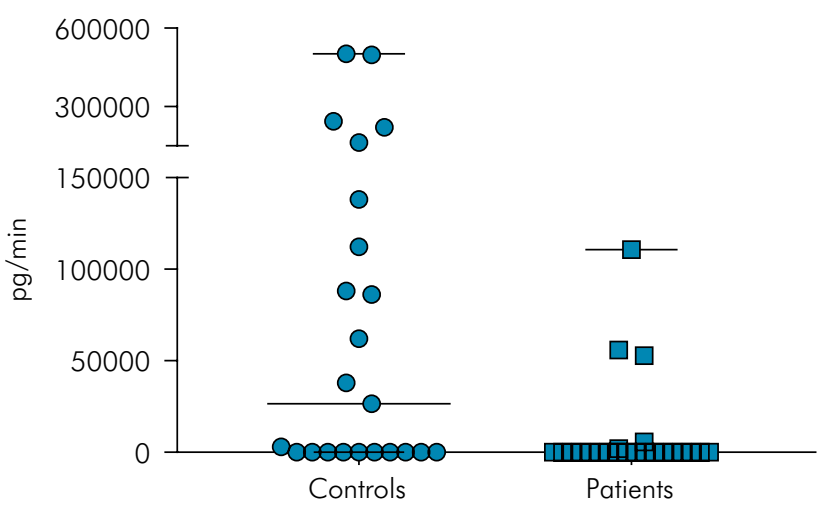

$p=0.0067$, Wilcoxon test

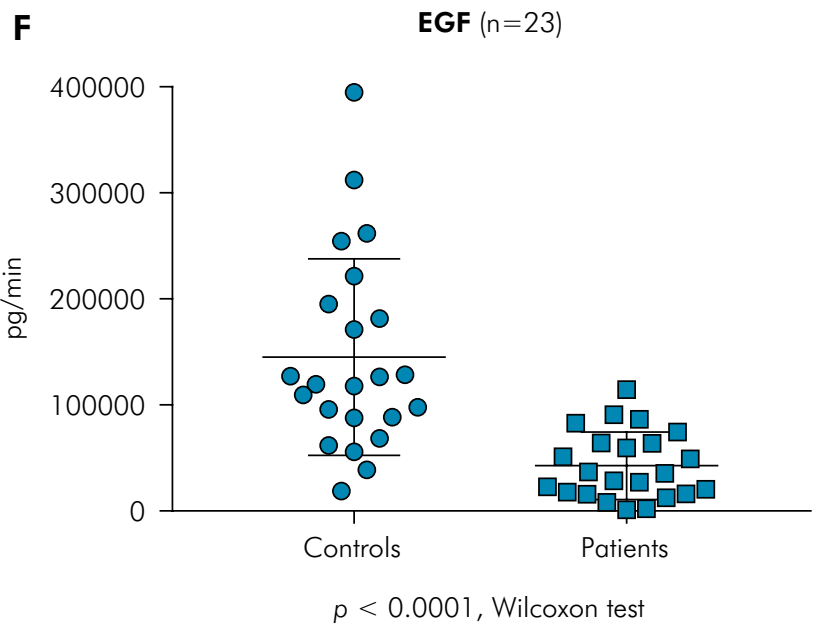

Figure 1. Comparison of salivary output $(\mathrm{pg} / \mathrm{min})$ of the receptor activator for nuclear factor $\kappa \mathrm{B}(\mathrm{RANK})$ and its ligand (RANK-L), osteoprotegerin (OPG), matrix metalloproteinase-9/ tissue inhibitor of metalloproteinase-2 (MMP-9/ TIMP-2), vascular endothelial growth factor (VEGF), and epidermal growth factor (EGF), between the controls and the patients irradiated in the head and neck region. 
Table 2. Spearman correlation coefficient $(\rho)$ among the salivary biomarkers in irradiated patients $(n=23)$.

\begin{tabular}{|c|c|c|c|c|c|}
\hline Variable & RANK & RANK-L & OPG & VEGF & MMP-9/ TIMP-2 \\
\hline RANK-L & +0.049 & & & & \\
\hline OPG & -0.039 & $+0.481^{*}$ & & & \\
\hline VEGF & +0.219 & $+0.746^{*}$ & $+0.548^{*}$ & & \\
\hline MMP-9/ TIMP-2 & +0.024 & +0.081 & -0.053 & $+0.500^{*}$ & \\
\hline EGF & +0.125 & $+0.894^{*}$ & $+0.469^{*}$ & $+0.792^{*}$ & +0.122 \\
\hline
\end{tabular}

*indicates $p<0.05$; " $"$ " indicates a negative correlation; " + " indicates a positive correlation. Modules between $0.1-0.3$ indicate a weak correlation; $0.4-0.6=$ moderate correlation; $0.7-0.9=$ strong correlation; $1.0=$ perfect correlation.

irradiated patients had lower levels of VEGF salivary output, which correlated with EGF and RANK-L, indicating that tissue vascularization, epithelial growth and bone remodeling processes may be altered after head and neck RT.

The reduced salivary EGF in irradiated patients described herein has been previously reported. ${ }^{24,25}$ Since most of the salivary EGF is derived from the acinar cells of the salivary glands, especially the parotid gland, ${ }^{26}$ its decrease in irradiated patients can be reasonably attributed to the RT-induced degeneration of the acinar structures. Some authors have reported a decrease in salivary EGF during RT treatment, and have correlated its decrease with a higher risk of oral mucositis during RT. 18,24,25,27,28 In the current study, the reduction in salivary EGF was seen to persist even after RT, thus highlighting that a relevant long-lasting alteration occurred in the saliva composition. This is of great importance considering the pivotal protective role of EGF in maintaining the oral mucosa. In contrast, Russo et al. ${ }^{17}$ reported a significant increase in salivary EGF and VEGF after RT, but only patients with oropharyngeal cancer were included in their study, and the biomarkers were assessed 12 months after RT.

The salivary alterations shown in the current study seem to be long-lasting RT-related side effects, since there was no difference based on RT completion time. Thus, the results seem to be unsupportive of establishing the best time after RT to perform invasive bone procedures in these patients. ${ }^{29,30}$ Instead, this corroborates these patients' need for regular and special oral care after RT. ${ }^{5}$ Finally, RT with adjuvant chemotherapy did not seem to influence the salivary biomarkers to any great extent, compared with RT without chemotherapy, insofar as the differences were observed only for VEGF and MMP-9/TIMP-2. This result should be interpreted with caution due to the small sample size in each group.

Some limitations can be drawn regarding this study. The occurrence of hyposalivation in the irradiated patients limits saliva sampling, and resulted in the exclusion of patients. This characteristic would limit the use of saliva as a diagnostic / predictive tool in such patients. Another limitation was the evaluation of biomarkers at a single time point after RT. Longitudinal studies evaluating the dosage of these biomarkers before, during and after RT treatment would contribute greatly toward defining this issue. Future studies should evaluate the predictive value of these biomarkers in the development, severity, and response to treatment of late complications of radiation therapy in the oral cavity.

\section{Conclusion}

In conclusion, patients submitted to head and neck radiotherapy had a reduced output of salivary molecules, which are of great importance for bone remodeling and tissue repair, namely RANK, RANK-L, MMP-9/ TIMP-2, VEGF, and EGF. The reduction in these molecules may disrupt tissue homeostasis, an issue that warrants further research to explore their role in the pathogenesis of the deleterious effects of radiotherapy in the oral cavity.

\section{Acknowledgments}

This study was supported by the Conselho Nacional de Desenvolvimento Científico (CNPq) [grant numbers 405585/2018-7, 302157/2017-4]; Pró-Reitoria de Pesquisa 


\author{
UFMG/ Fundação de Amparo à Pesquisa do Estado \\ de Minas Gerais (PRPq-UFMG/ PROBIC-FAPEMIG) \\ [grant number 04/2018]; FAPEMIG [grant number
}

\author{
APQ-01700-16]; Coordenação de Aperfeiçoamento \\ de Pessoal de Nível Superior (CAPES) [grant number \\ Financial code 001].
}

\section{References}

1. Marur S, Forastiere AA. Head and neck squamous cell carcinoma: update on epidemiology, diagnosis, and treatment. Mayo Clin Proc. 2016 Mar;91(3):386-96. https://doi.org/10.1016/i.mayocp.2015.12.017

2. Cooper JS, Fu K, Marks J, Silverman S. Late effects of radiation therapy in the head and neck region. Int J Radiat Oncol Biol Phys. 1995 Mar;31(5):1141-64. https://doi.org/10.1016/0360-3016(94)00421-G

3. Cheriex KC, Nijhuis TH, Mureau MA. Osteoradionecrosis of the jaws: a review of conservative and surgical treatment options. J Reconstr Microsurg. 2013 Feb;29(2):69-75. https://doi.org/10.1055/s-0032-1329923

4. Nadella KR, Kodali RM, Guttikonda LK, Jonnalagadda A. Osteoradionecrosis of the jaws: clinico-therapeutic management: a literature review and update. J Maxillofac Oral Surg. 2015 Dec;14(4):891-901. https://doi.org/10.1007/s12663-015-0762-9

5. National Institute of Health. PDQ ${ }^{\circledR}$ Supportive and Palliative Care Editoral Board. Oral complications of chemotherapy and head/neck radiation. Bethesda: National Cancer Institute; 2016 [cited year Month day]. Available from: https://www.cancer.gov/about-cancer/ treatment/side-effects/mouth-throat/oral-complications-hp-pdq

6. Asikainen PJ, Dekker H, Sirviö E, Mikkonen J, Schulten EA, Bloemena E, et al. Radiation-induced changes in the microstructure of epithelial cells of the oral mucosa: a comparative light and electron microscopic study. J Oral Pathol Med. 2017 Nov;46(10):1004-10. https://doi.org/10.1111/jop.12639

7. Liu C, Walter TS, Huang P, Zhang S, Zhu X, Wu Y, et al. Structural and functional insights of RANKL-RANK interaction and signaling. J Immunol. 2010 15;184(12):6910-9. https://doi.org/10.4049/jimmunol.0904033

8. Hanada R, Hanada T, Sigl V, Schramek D, Penninger JM. RANKL/RANK-beyond bones. J Mol Med (Berl). 2011 Jul;89(7):647-56. https://doi.org/10.1007/s00109-011-0749-z

9. Al-Dasooqi N, Gibson RJ, Bowen JM, Keefe DM. Matrix metalloproteinases: key regulators in the pathogenesis of chemotherapy-induced mucositis? Cancer Chemother Pharmacol. 2009 Jun;64(1):1-9. https://doi.org/10.1007/s00280-009-0984-y

10. Paiva KB, Granjeiro JM. Matrix metalloproteinases in bone resorption, remodeling, and repair. Prog Mol Biol Transl Sci. 2017;148:203-303. https://doi.org/10.1016/bs.pmbts.2017.05.001

11. Upile T, Jeries W, Kafas P, Harini S, Singh SU, Guyer M, et al. Salivary VEGF: a non-invasive angiogenic and lymphangiogenic proxy in head and neck cancer prognostication. Int Arch Med. 2009 Apr;2(1):12. https://doi.org/10.1186/1755-7682-2-12

12. Hu K, Olsen BR. The roles of vascular endothelial growth factor in bone repair and regeneration. Bone. 2016 Oct;91:30-8. https://doi.org/10.1016/i.bone.2016.06.013

13. Cohen S, Ushiro H, Stoscheck C, Chinkers M. A native 170,000 epidermal growth factor receptor-kinase complex from shed plasma membrane vesicles. J Biol Chem. 1982 Feb;257(3):1523-31. https://doi.org/10.1016/S0021-9258(19)68224-4

14. Berlanga-Acosta J, Gavilondo-Cowley J, López-Saura P, González-López T, Castro-Santana MD, López-Mola E, et al. Epidermal growth factor in clinical practice - a review of its biological actions, clinical indications and safety implications. Int Wound J. 2009 Oct;6(5):331-46. https://doi.org/10.1111/j.1742-481X.2009.00622.x

15. Vidotto A, Henrique T, Raposo LS, Maniglia JV, Tajara EH. Salivary and serum proteomics in head and neck carcinomas: before and after surgery and radiotherapy. Cancer Biomark. 2010-2011-2011;8(2):95-107. https://doi.org/10.3233/CBM-2011-0205

16. Laheij AM, Rasch CN, Brandt BW, de Soet JJ, Schipper RG, Loof A, et al. Proteins and peptides in parotid saliva of irradiated patients compared to that of healthy controls using SELDI-TOF-MS. BMC Res Notes. 2015 Nov;8(1):639. https://doi.org/10.1186/s13104-015-1641-7

17. Russo N, Bellile E, Murdoch-Kinch CA, Liu M, Eisbruch A, Wolf GT, et al. Cytokines in saliva increase in head and neck cancer patients after treatment. Oral Surg Oral Med Oral Pathol Oral Radiol. 2016 Oct;122(4):483-490.el. https://doi.org/10.1016/i.oooo.2016.05.020

18. Oton-Leite AF, Silva GB, Morais MO, Silva TA, Leles CR, Valadares MC, et al. Effect of low-level laser therapy on chemoradiotherapy-induced oral mucositis and salivary inflammatory mediators in head and neck cancer patients. Lasers Surg Med. 2015 Apr;47(4):296-305. https://doi.org/10.1002/lsm.22349

19. Rivero JA, Shamii O, Kolokythas A. Osteoradionecrosis: a review of pathophysiology, prevention and pharmacologic management using pentoxifylline, $\alpha$-tocopherol, and clodronate. Oral Surg Oral Med Oral Pathol Oral Radiol. 2017 Nov; 124(5):464-71. https://doi.org/10.1016/i.0ooo.2017.08.004 
Salivary molecules of bone remodeling and tissue repair after head and neck radiotherapy

20. Ortega N, Behonick D, Stickens D, Werb Z. How proteases regulate bone morphogenesis. Ann N Y Acad Sci. 2003 May;995(1):109-16. https://doi.org/10.1111/j.1749-6632.2003.tb03214.x

21. Nyman JS, Lynch CC, Perrien DS, Thiolloy S, O'Quinn EC, Patil CA, et al. Differential effects between the loss of MMP-2 and MMP-9 on structural and tissue-level properties of bone. J Bone Miner Res. 2011 Jun;26(6):1252-60. https://doi.org/10.1002/jbmr.326

22. Stegen S, Gastel N, Carmeliet G. Bringing new life to damaged bone: the importance of angiogenesis in bone repair and regeneration. Bone. 2015 Jan;70:19-27. https://doi.org/10.1016/j.bone.2014.09.017

23. Xu WL, Ong HS, Zhu Y, Liu SW, Liu LM, Zhou KH, et al. In Situ release of VEGF enhances osteogenesis in 3D porous scaffolds engineered with osterix-modified adipose-derived stem cells. Tissue Eng Part A. 2017 May;23(9-10):445-57. https://doi.org/10.1089/ten.tea.2016.0315

24. Epstein JB, Emerton S, Guglietta A, Le N. Assessment of epidermal growth factor in oral secretions of patients receiving radiation therapy for cancer. Oral Oncol. 1997 Sep;33(5):359-63. https://doi.org/10.1016/S1368-8375(97)00009-2

25. Dumbrigue HB, Sandow PL, Nguyen KH, Humphreys-Beher MG. Salivary epidermal growth factor levels decrease in patients receiving radiation therapy to the head and neck. Oral Surg Oral Med Oral Pathol Oral Radiol Endod. 2000 Jun;89(6):710-6. https://doi.org/10.1067/moe.2000.106343

26. Ino M, Ushiro K, Ino C, Yamashita T, Kumazawa T. Kinetics of epidermal growth factor in saliva. Acta Otolaryngol Suppl. 1993;500 sup500:126-30. https://doi.org/10.3109/00016489309126195

27. Epstein JB, Gorsky M, Guglietta A, Le N, Sonis ST. The correlation between epidermal growth factor levels in saliva and the severity of oral mucositis during oropharyngeal radiation therapy. Cancer. $2000 \mathrm{Dec} ; 89(11): 2258-65$. https://doi.org/10.1002/1097-0142(20001201)89:11<2258::AID-CNCR14>3.0.CO;2-Z

28. Normando AG, Rocha CL, Toledo IP, Figueiredo PTS, Reis PE, Canto GL, et al. Biomarkers in the assessment of oral mucositis in head and neck cancer patients: a systematic review and meta-analysis. Support Care Cancer. 2017 Sep;25(9):2969-88. https://doi.org/10.1007/s00520-017-3783-8

29. Kuo TJ, Leung CM, Chang HS, Wu CN, Chen WL, Chen GJ, et al. Jaw osteoradionecrosis and dental extraction after head and neck radiotherapy: A nationwide population-based retrospective study in Taiwan. Oral Oncol. 2016 May;56:71-7. https://doi.org/10.1016/i.oraloncology.2016.03.005

30. Wang TH, Liu CJ, Chao TF, Chen TJ, Hu YW. Risk factors for and the role of dental extractions in osteoradionecrosis of the jaws: A national-based cohort study. Head Neck. 2017 Jul;39(7):1313-21. https://doi.org/10.1002/hed.24761 